$\Rightarrow$ IMMUNE HOMEOSTASIS

\title{
Regulatory ILCs don't rely on FOXP3
}

Innate lymphoid cells (ILCs) have been subdivided into three main groups based on their similarity (in terms of transcription factor expression and cytokine production) to Thelper $1\left(\mathrm{~T}_{\mathrm{H}} 1\right), \mathrm{T}_{\mathrm{H}} 2$ and $\mathrm{T}_{\mathrm{H}} 17$ cells. To date, no ILC equivalent of a regulatory $\mathrm{T}\left(\mathrm{T}_{\mathrm{reg}}\right)$ cell has been identified, but Wang et al. now describe a unique subset of ILCs that produces regulatory-type cytokines.

ILCs are identified on the basis of their expression of CD45 and CD127 (the IL-7 receptor $\alpha$-chain) and their lack of expression of other lineageassociated markers ( $\left.\operatorname{Lin}^{-}\right)$. Wang et al. used reporter mice to identify a subset of $\mathrm{Lin}^{-} \mathrm{CD} 45^{+} \mathrm{CD} 127^{+}$ILCs that constitutively express the regulatory cytokine IL-10 in the intestine. IL-10producing ILCs were found in the lamina propria of the small intestine and colon, as well as in the small intestinal epithelium. Notably, unlike $\mathrm{T}_{\text {reg }}$ cells, IL-10-producing ILCs did not express the transcription factor FOXP3. They also did not express markers associated with the ILC1, ILC2 or ILC3 subsets. Similar IL-10producing ILCs were identified in human intestinal biopsies, and the authors propose that these cells represent a unique subset of ILCs, which they have named 'ILCregs'.

Transcriptome analyses confirmed that ILCregs do not express transcription factors associated with $\mathrm{T}_{\text {reg }}$ cells and other ILC subsets, such as FOXP3, T-bet, GATA3 and ROR $\gamma \mathrm{t}$. However, they constitutively express the transcription factors ID2, ID3 and SOX 4 as well as components of the receptors for IL-2 and transforming growth factor- $\beta$ (TGF $\beta$ ). Experiments to determine the origin of ILCregs showed that they arise from ID2dependent common helper-like innate lymphoid precursors and that they also require ID3 for their development and/or maintenance.

Importantly, the authors found that ILCreg populations expand during innate intestinal inflammation, secrete IL-10 and TGF $\beta$ and contribute to the resolution of the inflammatory response. The co-transfer of ILCregs protected mice in a model of innate colitis induced by the transfer of activated ILC1s and ILC3s, whereas $\mathrm{T}_{\text {reg }}$ cells could not suppress ILC1- and ILC3-driven inflammation in this system. Finally, the authors found that autocrine TGF $\beta 1$ is required for the expansion and maintenance of ILCregs during intestinal inflammation. It will be interesting to determine whether ILCregs are important for regulating innate inflammation in other tissues and disease settings. Yvonne Bordon

ORIGINAL ARTICLE Wang, S. et al. Regulatory innate lymphoid cells control innate intestinal inflammation. Cell http://dx.doi.org/10.1016/ j.cell.2017.07.027 (2017) 